\title{
Erratum to: Diagnostic criteria for IgG4-related ophthalmic disease
}

Hiroshi Goto - Masayuki Takahira - Atsushi Azumi •

Japanese Study Group for IgG4-Related Ophthalmic Disease

Published online: 24 February 2015

(C) Japanese Ophthalmological Society 2015

Erratum to: Jpn J Ophthalmol (2015) 59:1-7

DOI 10.1007/s10384-014-0352-2

The correct name of the second author should be given as Masayuki Takahira, not Masahiro Takahira.

The online version of the original article can be found under doi:10.1007/s10384-014-0352-2.

H. Goto $(\bowtie)$

Department of Ophthalmology, Tokyo Medical University, 6-7-1 Nishishinjuku, Shinjuku-ku, Tokyo 160-0023, Japan e-mail: goto1115@tokyo-med.ac.jp

M. Takahira

Department of Ophthalmology, Kanazawa University,

Kanazawa, Japan

A. Azumi

Department of Ophthalmology, Kobe Kaisei Hospital, Kobe,

Japan 